\title{
La investigación educativa en el área de lenguas: un estudio de trabajos realizados por egresados de 6 maestrías en Tabasco
}

\author{
Educational research in the area of Modern languagues: a study \\ thesis done by graduates of 6 master degrees in Tabasco
}

Juana May Landero ${ }^{1, *}$ (1)
Ernestina Patricia de Dios Naranjo ${ }^{2,}$

Artículo Científico

recibido: 06 de agosto de 2018

aceptado: 15 de diciembre de 2018

${ }^{1}$ División Académica de Educación y Artes, Universidad Juárez Autónoma de Tabasco. Av. Universidad s/n, Zona de la Cultura, Col. Magisterial, Vhsa, Centro, Tabasco, Mex. E-mail: educationphd2017@gmail.com

²División Académica de Educación y Artes, Universidad Juárez Autónoma de Tabasco. Av. Universidad s/n, Zona de la Cultura, Col. Magisterial, Vhsa, Centro, Tabasco, Mex. E-mail: patydn85@hotmail.com

*Autor de correspondencia: tionphd2017@gmail.com

\section{RESUMEN}

A continuación se presentan resultados de una investigación de corte cuantitativo sobre la Investigación Educativa (IE) en lenguas. Son datos derivados de una muestra aleatoria simple de 106 investigaciones, con diferentes géneros textuales, realizadas por egresados de 6 maestrías, de 3 instituciones públicas de Tabasco. El estudio tomó como referente la "ficha analítica y de clasificación de trabajos" del Consejo Mexicano de Investigación Educativa (COMIE), ajustada con categorías y subcategorías asociadas a los estudios de lenguas (lengua materna, lenguas extranjeras y lenguas indígenas), a partir del análisis de las investigaciones procedentes de las normativas de titulación, los enfoques metodológicos desarrollados, las temáticas abordadas y agrupadas en 12 categorías conceptualmente construidas. Esto con el fin de caracterizar lo que se ha realizado en este campo en Tabasco del que no se tienen registros y generar una fuente de consulta que sugiera las áreas de oportunidades para próximas investigaciones.

Palabras clave: Investigación educativa, lenguas, egresados.

\section{ABSTRACT}

The results of a quantitative research on educational research (EI) about languages are presented in this work.
The data from it was derived from a simple random sample of 106 investigations in which different text genres had been used. The reseaches were made by graduates of 6 masters, from 3 public institutions from Tabasco. The study took as reference the "analytical and job classification file" of the Mexican Council of Educational Research (COMIE). The data was adjusted into categories and subcategories and took into account that they were language studies (mother tongue, foreign languages and indigenous languages). In total they were grouped into 12 conceptually constructed categories. All of the researches were based on the degree regulations, the methodological approaches and themes addressed. The purpose of this work was to characterize research that has been done in the languages field in Tabasco because no records were found and also, to generate a source of consultation that can suggest areas of opportunity for future research.

Key words: educational research, languages, graduates.

\section{INTRODUCCIÓN:}

La Investigación Educativa (IE) ha trazado el camino para la reflexión y comprensión de los significados e intenciones de los sujetos que intervienen en la educación. Arias (2010), señala que, la IE es: "horizonte abierto y, por tanto, sumamente amplio, complejo, inacabado y difícil de abarcar porque está ligado a factores humanos, históricos y culturales en constante dinamismo, es el horizonte de diálogo a partir de los productos que se generan", (por lo que) "hay que asumirla como la memoria del quehacer científico sobre la educación", Weiss (2003).

En Tabasco la mayoría de las Instituciones de Educación Superior (IES) no cuentan con centros de documentación; no hay la cultura y las condiciones institucionales para la difusión y la divulgación. Sin ellas, es imposible que las comunidades académicas y científicas conozcan 
las contribuciones y hallazgos de sus pares, y no hay la oportunidad de que la sociedad comprenda y aproveche los beneficios del progreso científico.

Existe muy poca información clasificada y sistematizada sobre el conjunto de investigaciones educativas realizadas. No hay registros que den cuenta de la productividad científica en el campo de lenguas realizadas por docentes, egresados de las maestrías públicas de Tabasco. Por ello, surgen las siguientes interrogantes ¿Cuáles son los géneros textuales en que se presentan los trabajos de investigación con fines de titulación? ¿Qué temáticas se han plantado y con qué enfoque metodológico se han desarrollado? ¿Se han realizado investigaciones en el campo de lenguas? ¿Quiénes la han realizado? ¿Qué tipo de investigación realizan los docentes y en qué nivel educativo?

\section{DESARROLLO}

En las dos últimas décadas, las IES públicas y privadas han manifestado un mayor interés en la formación y profesionalización del profesorado ofreciendo maestrías encaminadas a promover una nueva cultural profesional, que ayude a superar los roles técnicos y operativos del magisterio y a reconfigurar el rol docente como facilitador, gestor y mediador de los asuntos pedagógicos. Otras, potencian la auto-reflexión, el análisis de las necesidades del alumnado, la participación en procesos de intervención, innovación y cambios en la escuela, así como el desarrollo de habilidades investigativas para la resolución de problemas y la producción de conocimientos en el aula.

\section{Las IES públicas en Tabasco y los programas de maestría}

De las 52 IES en Tabasco 29 (55.76\%) son públicas y 23 privadas (44.23\%); en ellas se ofrecen 92 Programas Educativos (PE) de maestrías: 51 en las IES públicas y 41 en las privadas. Con propósitos institucionales diferentes hay claras diferencias sobre la noción y práctica de la investigación. La mayoría de los PE tienen el carácter de "profesionalizante", en tanto que se orientan al dominio de lo disciplinar y a la mejora de la docencia, mayormente estas se imparten en las IES privadas. Los PE de maestría, cuyo propósito es el desarrollo y práctica de la investigación, están en las IES públicas. "En ellas se propicia la formación para la investigación mediante aprendizajes en el campo de los conocimientos, las habilidades, los hábitos, las actitudes y los valores, ... aprendizajes en el desarrollo de habilidades investigativas en torno a las cuales se ha producido poco conocimiento", Moreno (2003).

Algunos programas de maestrías en Tabasco ofertan titulación por créditos de estudios de doctorado y la automática por promedio que se reflejan en el índice de eficiencia terminal, pero no contribuyen a la práctica de la investigación. De acuerdo con datos de la SETAB, de las 23 IES privadas con registro ante la Secretaría de Educación de Tabasco (Cfr. SETAB, 2015), sólo 5 PE de maestrías promueven la titulación a través de tesis o algún trabajo de investigación.

Las IES en el país incluyen como una de sus funciones sustantivas la investigación. Es tema transversal que se observa en los planes y programas de estudio tanto a nivel licenciatura como en posgrado. Algunos están alineados a la política pública de fomento a la calidad del posgrado y son reconocidos dentro del Programa Nacional de Posgrado de Calidad (PNPC), (CONACYT, 2017). En tal contexto el quehacer investigativo adquiere mayor relevancia en su concepción y práctica. No es lo mismo "formar para la investigación a quien se dedicará a la investigación, que a quien necesita dicha formación para un mejor desempeño en su práctica profesional, o como herramienta para comprender y en su caso aplicar productos de investigación que le permitan resolver problemas", señala, Bayardo (2005).

\section{La investigación y las opciones de titulación en tres IES de Tabasco.}

De los 6 programas de maestría en el que se centra el análisis de este trabajo ninguno forma parte del PNPC, pero curricularmente se observan Líneas, áreas, asignaturas y/o seminarios de investigación educativa, en la que los estudiantes deben realizar investigación con fines de titulación. Ej. El Plan de estudios de la Maestría en Docencia del Instituto de Educación Superior del Magisterio de Tabasco, (Plan de estudios de M. en Docencia, IESMA, SETAB, 2000). Veamos que dice la normatividad.

\section{a. Opciones de titulación de las maestrías de la UJAT}

El Reglamento de titulación para los posgrados de la UJAT ha diversificado sus opciones de titulación de acuerdo con la orientación de sus programas y el ámbito disciplinar, persiste el interés institucional y académico del fomento a la investigación y a la titulación por tesis, (Reglamento de Posgrado en la UJAT, 2013). 
Perspectivas Docentes Vol.29 Núm. 66

En el caso de la Maestría en Educación, la cual estaba curricularmente orientada a la investigación, el Plan de estudios incluyó 4 asignaturas de Investigación educativa, en los cuales había que plantear un proyecto de investigación, desarrollarlo y presentarlo al término de la acreditación del último curso; una presentación en la modalidad de tesis en el proceso de titulación (Plan de estudios de la Maestría en Educación de la UJAT, 1996).

Sobre la Maestría en Docencia, el Plan de estudio se orientó a la profesionalización de docentes de todos los niveles educativos, y para ello, se encausaban metodologías cualitativas con fines de intervención didáctica, al término del último seminario de investigación el informe de resultados se convertía en un texto con formato de tesina con fines de titulación, (Maestría en Docencia de la UJAT, 2000).

\section{b. Opciones de titulación de la Universidad Pedagógica Nacional (UPN-271)}

La universidad Pedagógica Nacional (UPN) es una IES especializada en temas educativos en general y ofrece servicios a los maestros de educación básica y media superior en particular; ha definido entre sus prioridades la investigación..., desarrollando proyectos en temas emergentes de la problemática educativa, (Programa Integral de Desarrollo Institucional (PIDI) UPN 2014-2018). Alineada a la política educativa de formación de los nuevos cuadros docentes y a la profesionalización del magisterio en general, la UPN-271 ha diversificado su oferta educativa. Actualmente ofrece las maestrías en: Educación (la de mayor antigüedad), Gestión e Integración Educativa y Educación Media Superior.

En su Reglamento general para estudios de posgrado (2003), en el Cap. I. Art. 2 señala que el "objeto de los estudios de posgrado estará encaminado a la formación de profesionales de alta calidad, que amplíen, profundicen y apliquen el conocimiento en el campo de la educación, con la finalidad de satisfacer y propiciar el desarrollo integral del país en materia educativa" y que "la tesis de maestría o documento recepcional deberá ser un trabajo escrito e individual producto de la actividad académica desarrollada, en que demuestre el dominio del estudio del objeto seleccionado", Cap. II- Art. 36, Indicación II. De los anterior se deduce, el fuerte impulso a la investigación desde los programas de posgrado y el desarrollo de la misma en formato de tesis con fines de titulación.

\section{c. Opciones de titulación del Instituto de Educación Su- perior del Magisterio (IESMA).}

EI IESMA es una IES pública que ofrece servicios al magisterio que responde a la demanda que hacen los docentes de educación básica por estudios de maestría. Aquí se imparten las maestrías en Docencia y en Educación Especial con Enfoque Inclusivo, las cuales incluyen asignaturas relacionadas con el fomento a la investigación, (Plan de estudios de la M. en Docencia, IESMA, 2000). El reglamento de titulación de posgrado (2007) establece las siguientes modalidades: Memoria o Informe Académico por Actividad Profesional, Proyectos de innovación, aplicado o de intervención educativa, Tesis, Informe por la elaboración comentada de material didáctico para apoyar la docencia, Artículo académico, Por promedio y ensayo y Estudios de doctorado.

De lo anterior se infiere un interés institucional por promover la investigación, así como una noción común de la misma "como un proceso de construcción de conocimiento cuya dinámica demanda en forma conti-nua, pero diferenciada, la intervención de diversos procesos de orden cognitivo; esto es, procesos relacionados con la acción y el efecto de conocer", Moreno (2003). Asimismo, de concretarla en alguna de las opciones de titulación para lograr el grado del master estudiado, siendo la tesis el género textual que predomina, seguida de la tesina y artículos publicado, (ver gráfica No. 4). En el programa de la maestría en Docencia de la UJAT se establece que el género textual sea la tesina. Por el contrario, el IESMA considera diversas modalidades como: tesis, tesina, artículo publicado, estudios por doctorado y ensayo derivados de procesos de investigación.

\section{MATERIALES Y MÉTODOS}

Los datos que se presentan, son preliminares y corresponden a la descripción de datos empíricos de enfoque cuantitativo donde se da cuenta de la producción investigativa en el área de lenguas que para fines de titulación realizaron (docentes) egresados de 6 programas de maestría en Tabasco, del 2005 al 2015.

El corpus de análisis lo constituyen 106 investigaciones con diferentes géneros textuales que resultan de un muestreo aleatorio simple. Trabajos con temáticas relacionadas con el campo de las lenguas, es decir, documentos donde se analizan temáticas relacionadas con la Lengua española como lengua materna; de inglés, italiano 
y francés como lenguas extranjeras y del chontal- Yokot'an-, como lengua indígena. Dichos trabajos se encontraron en las bibliotecas y centros de documentación de 3 IES públicas de Tabasco (UJAT, IESMA y UPN-271).

Para la recogida de datos se tomó como base la "ficha analítica y de clasificación de trabajos" del COMIE, a la cual se le realizaron ajustes en las categorías y subcategorías de análisis a fin de incluir aspectos relacionados con el campo de lenguas. Para el registro se diseñó una base de datos en Excel con categorías y subcategorías para identificar y describir las características metodológicas y temáticas de nuestro interés, que se transfirieron a la aplicación SPSS-V22 para cuantificar los resultados.

\section{Hallazgos}

El corpus de trabajos de investigaciones en lenguas realizadas por docentes de diferentes niveles educativos, egresados de 6 diferentes Programas de Maestrías de IES públicas de Tabasco, fue de 106 documentos con diferentes géneros textuales y con fines de titulación.

En las maestrías en Educación, Docencia y Enseñanza del Inglés de la UJAT fueron 36; en Docencia y Educación Especial con enfoque Inclusivo del IESMA, 8; en las de Educación y Docencia de la UPN-271, 62. Destacar que, de los 36 trabajos de la UJAT, sólo se identificaron 2 de la maestría en la Enseñanza del Inglés, lo que refleja un índice muy bajo en la eficiencia terminal y la poca aportación investigativa de este programa al campo de las lenguas. Respecto a la UPN, de ella tenemos el mayor número de trabajos que conforman el corpus de nuestra investigación. La Figura 1 nos describe con precisión el índice de trabajos encontrados por institución.

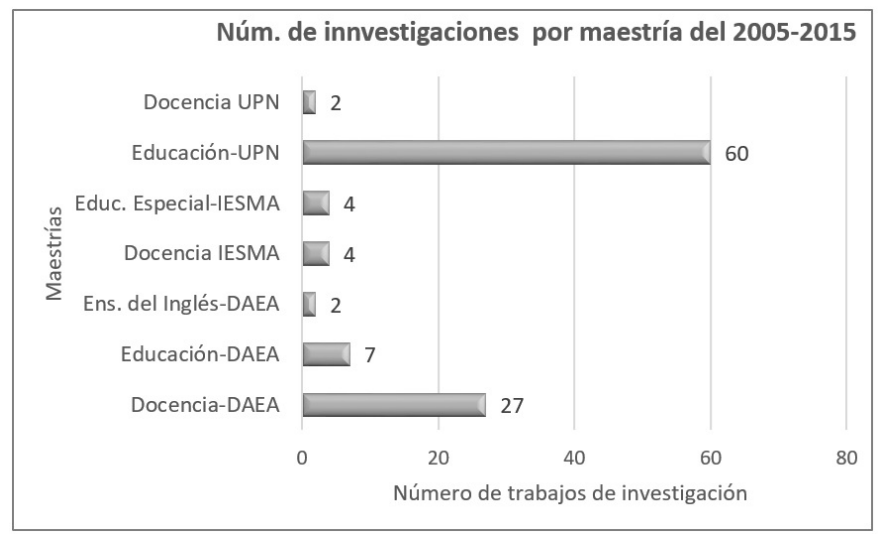

Figura 1. Número de investigaciones encontradas por programa de maestría y por instituciones
Respecto a la procedencia de los autores de las investigaciones, podemos afirmar que el $100 \%$ son docentes que proceden de diferentes niveles educativos. Ochenta y tres de los 106 trabajos fueron realizados por docentes de educación básica ( 6 de preescolar, 59 de primaria y 8 de secundaria-4 de telesecundaria-) lo cual equivale al $78.3 \%$, mientras que 8 fueron hechos por profesores de educación media superior y 14 de licenciatura. Así mismo, se observa que los 106 trabajos se realizaron en el nivel educativo donde trabaja el autor. Por ejemplo, la maestría de preescolar aborda un tema para favorecer la lecto escritura en su grupo de 3ero. de preescolar. Esto significa un paso importante en el proceso investigativo. Primero porque, los temas abordados se identifican y se problematizan en la idea de analizar el problema y al mismo tiempo se asume una posición de intervención pedagógica, para lo que se construye una metodología en aras de la indagación. Segundo, por la actitud reflexivo que este proceso provoca sobre la actuación docente al convertirla en objeto de investigación.

La figura 2 indica que 83 de los docentes proceden de educación básica (78.3\%): 59 de primaria, 18 de secundaria y 6 de preescolar; sólo 8 (7.54\%) de media superior y $14(13.2 \%)$ de licenciatura.

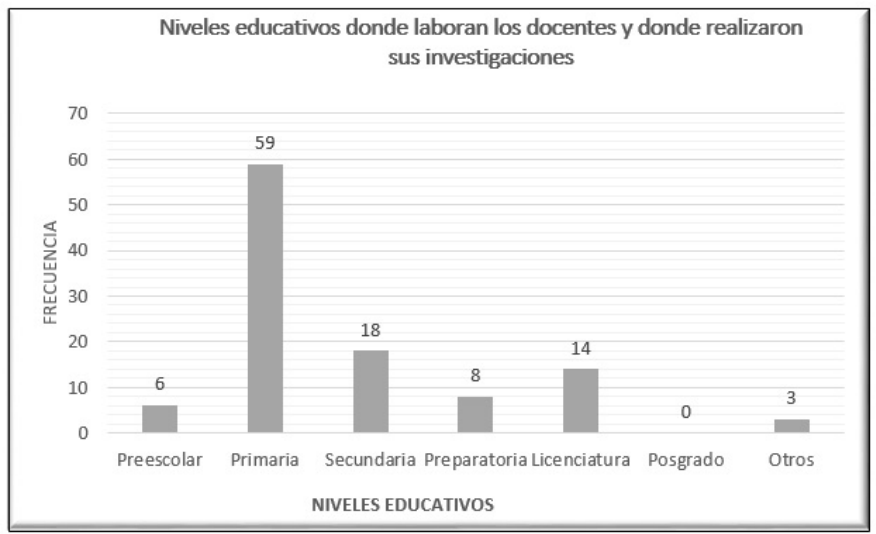

Figura 2. Niveles educativos de procedencia laboral de los docentes

Sobre el formato que tienen las investigaciones, se observa que el género textual en el que mayormente se presentó la producción investigativa, fue en la modalidad de tesis con el $87.7 \%$ (93 trabajos), el 10.8\% fueron tesinas (11) y 2 (1.8\%) artículos publicados.

Dado que la mayoría de los temas se ubican en el ámbito de lo pedagógico, hay una correlación entre la temática y el enfoque metodológico en el desarrollo de la investigación. Es decir, 71 proyectos son de intervención pe- 
Perspectivas Docentes Vol.29 Núm. 66 La investigación educativa en el área de lenguas May Landero / de Dios Naranjo

dagógica, que suponen la reflexión sobre la propia práctica escolar, y en 76 se desarrollaron desde un enfoque cualitativo. De acuerdo con González, Nella, et al (2007), "la reflexión es el mejor instrumento para un aprendizaje significativo, para un genuino desarrollo profesional y para iniciar el camino hacia el cambio. Cuando el docente investiga en y sobre su acción, se convierte en un investigador en el aula en su escuela y comunidad. No depende ya ni de técnicas, rutina, recetas, normas ni de prescripciones curriculares impuestas... depende fundamentalmente de sus propios descubrimientos, de la teoría que va elaborando en su hacer pedagógico reflexionado permanentemente". Esto significa un trabajo de análisis e interpretación de datos de una narrativa que recoge de manera textual lo expresado por los informantes mediante instrumentos de recogida de datos. Por otra parte, se encontró que el enfoque metodológico que predomina en los 106 trabajos de investigación fue el cualitativo con el $71.6 \%$, seguido del enfoque mixto en 17 y 13 en cuantitativo.

Para categorizar las temáticas que se incluyen en la Figura 3, inicialmente se analizaron los títulos para identificar variables, mismas que se relacionaron con los objetivos y preguntas de investigación, así como con los diseños metodológicos de cada investigación. Se leyeron 106 temáticas, se clasificaron con base a diferencias y similitudes conceptuales. Luego se reagruparon hasta lograr las 12 categorías en las que se organizaron; véase Figura 3. En él se observa la recurrencia de 4 importantes áreas relacionadas con el tema común de lenguas (lengua española, inglesa, italiana, francesa y chontal). I. "Estrategias para la enseñanza y aprendizaje de lenguas" con un 43.3\%. II. "Comprensión lectora" con el 17.9\% (19) y "Expresión escrita" con el 13.2\%.

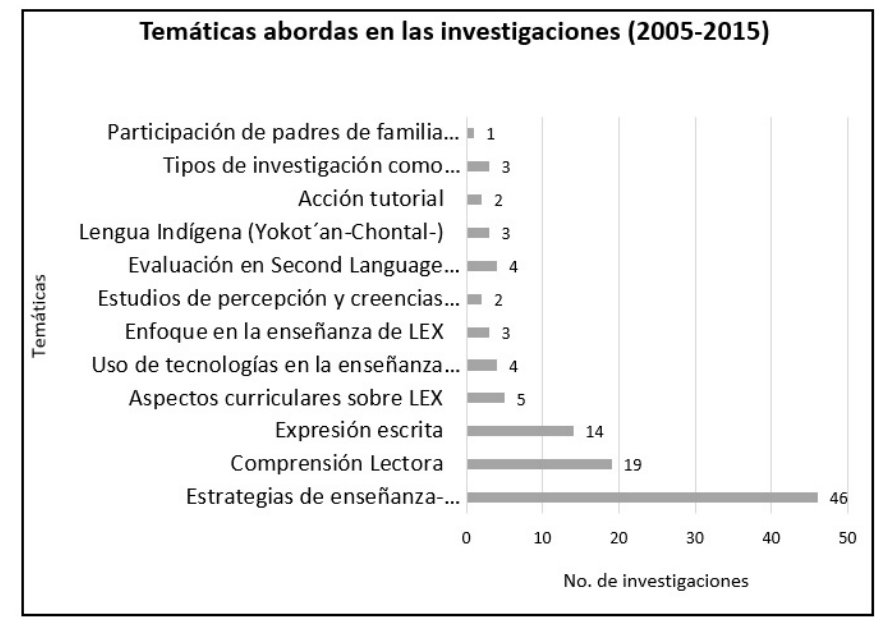

Fgura 3. Categorización de las temáticas abordadas en las investigaciones

\section{CONCLUSIONES}

Los datos presentados sobre la investigación y su relación con los procesos de titulación en los posgrados de Tabasco revelan, por una parte, el incipiente desarrollo de la investigación educativa en el campo de las lenguas. 106 trabajos realizados en un periodo de 10 años, equivale a 10.6 trabajos por año. Si eso lo dividimos entre las 6 maestrías seleccionadas tendríamos 1.7 trabajos de maestría por año. Datos poco significativos, que describen el bajo índice de eficiencia terminal de nuestras instituciones educativas y el bajo interés para investigar en este campo.

Si bien, la normativa de titulación es congruente con el interés por desarrollar la práctica de la investigación, es necesario que los procesos curriculares incluyan la diversificación metodológica y textual más allá de las modalidades planteadas. Seguramente la "tesis", por la integración y rigurosidad metodológica seguirá fomentándose en las comunidades académicas, en el posgrado; sin embargo, es necesario replantear los enfoques, tipos y diseños de investigación mas allá de los procesos de titulación, dando prioridad a la formación de habilidades y al desarrollo de la investigación desde otras modalidades y formatos, así como la de propiciar caminos de difusión y divulgación de los resultados.

De las temáticas, se identificaron las variables de análisis y se cotejaron con los diseños de investigación desarrollados. Se observó que en su mayoría fueron trabajos de intervención pedagógica y, por ende, el enfoque metodológico desarrollado fue el cualitativo con el $71.6 \%$; de estos el 33.9\% fueron estudios de caso; $15.09 \%$ investigación-acción participativa y el $2.8 \%$. Es decir, que son estudios de contextos específicos y análisis en micro de situaciones problemáticas que fueron intervenidas con propósitos de mejora y transformación de la realidad.

Con las variables de los 106 trabajos se organizaron y clasificaron las temáticas. De ese ejercicio resultaron 12 categorías, prevaleciendo el interés en 3 temas centrales del campo de lenguas: 1 . Estrategias de enseñanza aprendizaje de lenguas con un $43.3 \%$ (46 trabajos). II. Comprensión lectora con el 17.9\% (19). III. Expresión escrita con el $13.2 \%$ de 14 .

Estos datos constituyen una fuente valiosa para identificar las debilidades de la práctica investigativa en el campo de las lenguas y una extra-ordinaria oportunidad para 
Perspectivas Docentes Vol.29 Núm. 66 La investigación educativa en el área de lenguas May Landero / de Dios Naranjo

establecer áreas de oportunidades para encausar investigaciones posteriores. Asimismo, es una aportación valiosa al campo de la investigación en México y particularmente en Tabasco, al proporcionar datos y fechas de los pocos trabajados realizados y dispersos.

\section{REFERENCIAS}

Arias, Eduardo. (2012). La investigación educativa un horizonte abierto. Revista Electrónica de Educación: Sinéctica, (38), enero - junio. Universidad Jesuita de Guadalajara.

Consejo de Ciencia y Tecnología del Estado de Tabasco (2017).

Consejo Nacional de Investigación Educativa (2016).

González, Nella, et al (2007). La investigación educativa en el hacer docente. Revista de Educación Lurus, vol. 13 No. 23. Universidad Pedagógica Experimental Libertador, Caracas. Venezuela. Recuperado en: http://www.redalyc. org/pdf/761/76102315.pdf

International Bussines Machines [IBM Corp.] (2013). SPSS Software estadístico. Version 22.0. Armonk, NY.

Instituto de Educación Superior del Magisterio, (2000). Plan de estudios de la Maestría en Docencia-IESMA-SE$\mathrm{TAB}$

$\mathrm{TAB}$

(2007). Reglamento de titulación de Posgrado, SE-

Moreno, Bayardo María Guadalupe (2003). La propuesta de una formación para la investigación centrada en el desarrollo de habilidades. Educar: Revista de Educación, 26, julio-septiembre. Recuperado en: http:// www.quadernsdigitals.net/datos/hemeroteca/r_24/ nr_549/a_7674/7674.pdf

Moreno, Bayardo maría Guadalupe (2005). Potenciar la educación. Un curriculum transversal de formación para la investigación. Rev. Electrónica Iberoamericana sobre calidad, Eficacia y Cambio en Educación (REICE) Vol. 3, No. 1. Recuperado en: http://www.ice.deusto.es/rinace/ reice/vol3n1_e/Moreno.pdf
Universidad Autónoma de Tabasco [UJAT] (2013). "Reglamento de titulación de estudios de posgrado".

(1996). Plan de estudios de la Maestría en Educación, DAEA.

(2000) Plan y programas de estudios de la Maestría en Docencia, DAEA

Universidad Pedagógica Nacional (2003). Reglamento general para estudios de posgrado, SEP.

Universidad Pedagógica Nacional, (2014). Programa Integral de Desarrollo Institucional-UPN 2014-2018.

Weiss, Eduardo (2003) Coord. Vol. 1. El campo de la Investigación Educativa. En Colección: La Investigación Educativa en México 1992-2002, 1ạ. Ed. COMIE, AC.

\section{Semblanza de los autores}

${ }^{1} J u a n a$ May Landero: Licenciada en Ciencias de la Educación, Especialidad en Docencia, Maestra en Educación y Doctorante en Educación. Profesora-Investigadora de Tiempo Completo en asignaturas de investigación en la Licenciatura en Idiomas de la UJAT. Responsable y colaboradora de proyectos de investigación. Perfil PRODEP. Estudios sobre Lengua Inglesa en The University of Southern Mississippi en Hattiesburg, MS, Estados Unidos de Norteamérica y Ex becaria de la fundación Fullbright-García Robles en la Somerset High School, en Somerset, Pennsylvania, Estados Unidos de Norteamérica.

\footnotetext{
2.Ernestina Patricia de Dios Naranjo: Licenciada en Ciencias de la Educación egresada de la UJAT. Especialidad en la Enseñanza del Idioma Inglés de la Universidad Autónoma de Yucatán y la Universidad de Cambridge. Maestra en Lingüística Aplicada por la UNAM. Profesora de tiempo completo y fundadora de la Licenciatura en Idiomas de la UJAT. Líneas de investigación sobre enseñanza del idioma inglés, lingüística y análisis del discurso.
} 